\title{
El rol del docente de matemáticas en el desarrollo del pensamiento crítico en la enseñanza remota
}

\section{Happiness at work in official elementary and middle school teachers}

\author{
Sonia Valbuena-Duarte ${ }^{1}$ \\ Karen De La Hoz Coronado² \\ Jesús Berrio Valbuena ${ }^{3}$
}

\begin{abstract}
RESUMEN
Con este artículo se socializa un trabajo de investigación cuyo objetivo fue caracterizar la incidencia de las prácticas del docente de matemática en la habilidad del pensamiento crítico del estudiante. El método utilizado se enmarcó en un enfoque cualitativo, bajo un diseño de estudio de caso, con una muestra de dos docentes de una institución de la Región Caribe colombiana, la información fue recabada a través de la observación, cuestionarios, diario de campo y entrevistas. En los resultados obtenidos se evidenció que aun cuando el 1 Magíster en Educación: Desarrollo Humano, Magíster en Matemáticas, Docente investigadora tiempo completo, Grupo GIMED. Universidad del Atlántico, Puerto Colombia, Colombia. soniabalbuena@mail.uniatlantico.edu. co. https://orcid.org/0000-0003-3667-1087

$2 \quad$ Magister (est) en Educación. Docente Institución Secundaria, Barranquilla. nerak124@hotmail.com

$3 \quad$ Magíster en Educación Matemática. Docente investigador. Grupo GHEM. Universidad del Atlántico, Puerto Colombia, Colombia. jberriovalbuena@mail.uniatlantico. edu.co. http://orcid.org/0000-0002-4014-5322.
\end{abstract}

docente muestra algún conocimiento del pensamiento crítico, en su praxis no se logró hallar indicios de estrategias que lo promuevan en los estudiantes en el aula de clases, por lo que se diseñan tareas didácticas que se comparten con los docentes con el propósito de promover desarrollos de pensamiento crítico en los estudiantes en el área de matemática. Los resultados obtenidos son satisfactorios para este propósito. Por lo que se concluye que cuando el docente realiza una planeación considerando el desarrollo de esta habilidad en sus estudiantes se optimizan procesos de aprendizaje en el estudiante, por lo tanto, aplicar el diseño de estas secuencias didácticas en matemáticas posibilita la asimilación del aprendizaje y a la innovación por parte de los docentes de salir de la monotonía e implementar nuevas alternativas de participación de sus estudiantes buscando 
que esta sea activa y permanente durante la clase.

PALABRAS CLAVES: pensamiento crítico, práctica docente, habilidades, secuencias didácticas.

\section{ABSTRACT}

This article presents a research work that aims to characterize the incidence of the mathematics teacher's practices on the student's critical thinking ability. The method used was framed in a qualitative approach, under a case study design, with a sample of two teachers from an institution in the Colombian Caribbean Region, the information was collected through observation, questionnaires, field diary and interviews . In the results obtained it was evidenced that even when the teacher shows some knowledge of critical thinking, in his praxis it was not possible to find indications of strategies that promote it in the students in the classroom, so didactic tasks are designed that are shared with teachers in order to promote development of critical thinking in students in the area of mathematics. The results obtained are satisfactory for this purpose. Therefore, it is concluded that when the teacher makes a planning considering the development of this ability in their students, learning processes are optimized in the student, therefore, applying the design of these didactic sequences in mathematics enables the assimilation of learning and innovation on the part of teachers to get out of the monotony and implement new alternatives for the participation of their students, seeking to make it active and permanent during class.

KEYWORDS: critical thinking, teaching practice, skills, didactic sequences.

\section{RESUMO}

Com este artigo socializa-se um trabalho de pesquisa cujo objetivo foi caracterizar a incidência das práticas do professor de matemática na capacidade de pensamento crítico do aluno. O método utilizado com abordagem qualitativa, sob a forma de estudo de caso, com uma amostra de dois professores de uma instituição da Região do Caribe colombiano, as informações da pesquisa foram obtidas por meio de observação, questionários, diário de campo e entrevistas. Nos resultados obtidos evidenciouse que mesmo quando o professor demonstra algum conhecimento de pensamento crítico, em sua práxis não foi possível encontrar indícios de estratégias que promovam essa habilidade nos alunos em sala de aula, por isso são elaboradas tarefas didáticas que são Eles compartilham com professores com o objetivo de promover o desenvolvimento do pensamento crítico nos alunos da área de matemática. Os resultados obtidos são satisfatórios para este fim. Portanto, conclui-se que quando o professor faz um planejamento considerando o desenvolvimento dessa habilidade em seus alunos, os processos de aprendizagem são otimizados no aluno, portanto, a aplicação do desenho dessas sequências didáticas em matemática possibilita a assimilação da aprendizagem e inovação por parte dos professores para sair da monotonia e implementar novas alternativas de participação de seus alunos, buscando torná-la ativa e permanente durante as aulas.

PALAVRAS-CHAVE: pensamento crítico, prática docente, habilidades, sequências didáticas.

\section{INTRODUCCIÓN}

El pensamiento crítico (Ayola y Moscote, 2018; Febres et al., 2017; Camargo y Useche, 2015) entendido como una destreza que genera un grado mayor de la creatividad en el aula de clases favoreciendo una asimilación de conocimientos a través de un proceso de reflexión entre el saber y el quehacer que hacen parte del contexto; esta habilidad se va consolidando en el ambiente y espacio educativo, ya que a partir de esos momentos se crean las bases que iniciaran el proceso de pensar formalmente. De esta manera 
el pensamiento crítico es considerado como una gran estrategia que podría impactar de manera significativa los procesos de enseñanza y aprendizaje.

El rol que desempeña el profesor en el proceso educativo es altamente relevante pues es el diseñador del ambiente, por lo que se espera esté en continua formación que le fortalezca en la búsqueda, proposición, gestión e innovación de propuestas didácticas que permitan favorecer los aprendizajes en los estudiantes, promoviendo en estos la reflexión efectiva sobre sus propios aprendizajes (Caradonna, 2017; Parra,2014) es decir, el docente en la cotidianidad del aula se espera dirija su praxis de manera que se ejecute utilizando e implementando diferentes métodos que sirvan como mediadores para lograr desarrollar aprendizajes satisfactorios y de esta forma transformar la práctica educativa mediante la interacción que pueda establecer con sus estudiantes a partir de un saber especifico.

A los aprendizajes efectivos en matemáticas se les atribuye por naturaleza el aporte al desarrollo de habilidades en los individuos tales como interpretar, comprender el entorno, emitir juicios y validar resultados, autores tales como Rodríguez (2013) y López (2014) también lo relacionan con el desarrollo del pensamiento crítico para fomentar "un ciudadano autónomo"; de allí que desde la cotidianidad en el evento pedagógico, se observa que los estudiantes presentan serias dificultades, en proponer situaciones problemas al mismo tiempo que plantear y poder sustentar sus propias reflexiones obteniendo resultados pocos satisfactorios dentro de su proceso académico, así dentro de las variables que pudieran tener incidencia para este propósito llama la atención volver la mirada a las estrategias que el docente está utilizando dentro de su práctica para motivar a sus estudiantes a meditar frente a diferentes situaciones problemas y qué tanto estos están preparados para lograr incentivar el desarrollo del pensamiento crítico de los individuos, de esta manera se puede llegar a la construcción del conocimiento de una forma más clara, didáctica, espontanea permitiendo que en el proceso de enseñanza se puedan potencializar habilidades, destrezas y competencias matemáticas.

Gran parte de las investigaciones sobre desarrollo de pensamiento crítico y práctica docente se han centrado en los estudiantes (Núñez, Ávila, \& Olivares, 2017); en otros casos se han enfocado en estudiantes universitarios y la relación que se puede establecer entre este tipo de pensamiento para la vida académica y personal de los estudiantes (López, 2012; Gavilanes \& Benavides, 2018); de la misma manera (Yasaldez et al., 2018), plantean la importancia de desarrollar habilidades del pensamiento crítico como lo son analizar, inferir, solucionar problemas empleando estrategias que generan espacios de motivación, colaboración, autorregulación y cambio de forma de pensar de los estudiantes. Por lo anterior, estas investigaciones se han centrado en el desarrollo del pensamiento crítico en los estudiantes y han dejado a un lado la importancia del roll del docente en el proceso de enseñanza como mediador para desarrollar habilidades del pensamiento crítico en el aula de clases de sus estudiantes desde su práctica docente, interés central de la presente investigación.

Se selecciona un estudio de caso, dado que son dos docentes objetos de estudio en esta investigación y se quiere tener una información pertinente y un acompañamiento de ellos para lograr plantear estrategias que le faciliten el desarrollo del pensamiento crítico en sus estudiantes. Son motivaciones en esta investigación la búsqueda de respuestas a interrogantes tales como ¿De qué recursos, modelos y estrategias dispone el docente para poder constatar y revisar si su planeación de clase va en dirección con lo que quiere proyectar en el proceso de enseñanza? ¿Qué caracteriza 
las prácticas del docente de matemáticas para desarrollar el pensamiento crítico de los estudiantes?

\section{PENSAMIENTO CRÍTICO}

Existen diversas concepciones que se han planteado con relación al pensamiento crítico, sin embargo, no se ha definido un concepto formal sobre él, a continuación se establece relaciones entre los siguientes autores quienes consideran que el pensamiento crítico Arenas (2007); Paul y Elder (2004); Mackay et al. (2018); Aguilar et al. (2020) se refiere a una habilidad sumamente relevante para el ser humano, le permite a este discernir acertadamente en diferentes situaciones y aspectos como en la vida laboral, académica y cotidiana. Dicha habilidad le permite al individuo realizar sensaciones internas como la reflexión, la intuición y la construcción de juicios de valor que son emitidos por el interlocutor.

\section{HABILIDADES DEL PROCESO EN EL PENSAMIENTO CRÍTICO}

Las habilidades de pensamiento crítico deben ser estimuladas permanentemente, de esta forma de acuerdo a Cangalaya (2020); Frías et al. (2017); Campos (2020), manifiestan que la interpretación permite adquirir una perspectiva intensa de los conocimientos que se han logrado aplicándolos como información relevante generando sentido, juicios y significados para poder usarlos como evidencias válidas y consistentes. Análisis permite desintegrar en todas sus partes esenciales, es decir tratar de revelar nuevas relaciones y conexiones. Involucra a su vez la comparación de información, la capacidad de contrastarla con el propósito de expresar creencias, juicios u opiniones. La evaluación a través de esta habilidad se valora la credibilidad de los enunciados, representaciones; describiendo la percepción, experiencia o juicio de un individuo. La inferencia se refiere a cuestionar e identificar las evidencias para poder proponer nuevas alternativas y de esta manera sacar conclusiones. La explicación esta habilidad se refiere a la capacidad de argumentar y presentar los resultados propios de una idea de manea clara, reflexiva y coherente finalmente la autorregulación permite tener una visión general y amplia, que busca el modo más flexible y sin prejuicios, de ver una situación.

Reflexión de la praxis docente del docente de matemáticas

Es necesario señalar que dentro del proceso de enseñanza de las matemáticas es importante que el docente permanezca en una observación y evaluación constante de su praxis, de esta forma se permitirá conocer cuáles son los elementos que está utilizando en la implementación del proceso de enseñanza; así Arévalo (2015), Jiménez et al. (2016) aluden que el profesor debe tener en cuenta en la enseñanza ciertas destrezas y habilidades profesionales que le puedan proporcionar y ofrecer un espacio confortable que le pueda permitir una mejor interacción con sus estudiantes y poder lograr resultados en los aprendizajes de una manera dinámica y fluida a partir del contexto, las planeaciones y las necesidades propias de la comunidad.

La práctica de enseñar matemáticas puede contextualizarse en diversos momentos que van desde la planificación del evento pedagógico a momentos imprevistos durante el desarrollo de la clase Llinares (2019), esto debido a la responsabilidad que implica el manejo de diferentes estilos de aprendizaje de los estudiantes, ya que no existen estudiantes uniformes y como docentes gestores de cambio debemos encontrar los recursos adecuados para poder planificar sin pensar en los niveles de cada estudiante para que estos puedan trabajar determinado problema o situación. 


\section{METOdOLOGÍA}

Se realizó observación no participante durante el desarrollo de algunas clases impartidas y planificadas por los docentes. Posteriormente se efectuó una transcripción detallada para lograr adquirir de manera clara una forma textual de los datos utilizados, la cual fue empleada para generar una reflexión en la presente investigación. El enfoque de trabajo es cualitativo y el diseño investigativo utilizado fue un estudio de caso (Jiménez et al., 2016 y Alzora 2019). La metodología para desarrollar la investigación se desarrolló en cuatro fases:

En las fases 1 y 2 se seleccionó el caso del desarrollo del pensamiento crítico en el estudiante desde la práctica del docente de matemáticas, el cual es relevante ya que a través de este se logra conocer cómo se implementan las clases en el área de matemáticas en la búsqueda de potenciar el que hacer del docente dentro del aula y a partir de ellas potencializar las habilidades del pensamiento crítico presente en los estudiantes, dentro del diseño de instrumentos para recabar información se realizó un cuestionario socio demográfico y de preguntas abiertas para conocer las interpretaciones de los profesores con relación al pensamiento crítico y las diferentes estrategias utilizadas a lo largo de su carrera como docente, seguida de una entrevista y la observación no participante de la clase.

En las fases 3 y 4 se realizó el análisis de la información a partir de las observaciones en clases, de esta manera se identificó las habilidades del pensamiento crítico presente en los estudiantes en el desarrollo de cada una de la clases y de esta forma poder intervenir a partir de la ejecución de un conjunto de actividades con el fin de fortalecer el pensamiento crítico a través de la práctica orientada por el docente, de igual forma la información recolectada del docente permitió corroborar la ausencia de estrategias dentro del salón de clases reflejada en los resultados de los estudiantes. $\mathrm{Se}$ establecieron comparaciones entre las actuaciones, estrategias, implementación de tareas utilizadas por el profesor, se trianguló con base a los autores que soportan cómo se desarrolla las habilidades del pensamiento crítico en los estudiantes.

\section{POBLACIÓN Y MUESTRA}

La población objeto de estudio en esta investigación son los docentes de básica secundaria de una institución de carácter privado de la ciudad de Barranquilla caracterizada por ofrecer un sistema de educación formal el cual consiste en cursar y realizar el bachillerato en menos tiempo del convencional. Sin reducir el contenido académico ni la calidad educativa. Se clasifica de la siguiente forma: Ciclo 2:(4 y 5to Primaria) 1 año cada curso, Ciclo 3: ( 6 y $7 \mathrm{mo}$.) 1 año, ciclo 4: (8 y 9no.) 1 año, Ciclo 5: (10 Grado) 6 meses y Ciclo 6: $\left(11^{\circ}\right.$ Grado) 6 meses. La filosofía del Colegio es crear un vínculo positivo entre el estudiante y el aprendizaje busca transmitir el conocimiento de forma en que el estudiante lo adquiera con claridad, motivación, afecto y responsabilidad. El modelo pedagógico del colegio es el desarrollo de pensamiento crítico. Enseñarles a los estudiantes a que aprendan a analizar, interpretar y evaluar cualquier situación y/o conocimiento logrando adquirir juicio crítico con sentido común y discernimiento en valores. Desarrollar el pensamiento crítico permite tomar consciencia de los pensamientos para reflexionar y evaluarlos de forma efectiva.

El muestreo seleccionado es intencionado autores como Bonilla y Rodríguez (2005); consideran que es importante conformar una muestra que permita reflejar casos característicos de una población determinada, en donde se reflejan varios aspectos fundamentales y que cumplen las condiciones de estudio de esta investigación. 
La muestra está constituida por dos docentes del área de matemáticas de la básica secundaria de los grados $7^{\circ}$ y $9^{\circ}$, identificados en este estudio como Profesor 1 y Profesor 2. Los docentes en mención tienen pregrado en Física, sus edades oscilan entren 29 y 32 años de edad, ambos cuentan con una experiencia no mayor a 5 años en instituciones educativas, no ejercen su formación profesional en otros espacios diferentes a la docencia y no poseen estudios de posgrados. Para recopilar información se realizó una observación no participante, por ende, los desarrollos de las clases implementadas por los docentes fueron observadas y grabadas con el consentimiento de los actores, de la misma manera se tomaron apuntes en el diario de campo sin tener algún tipo de intervención.

\section{TÉCNICAS E INSTRUMENTOS PARA LA RECOLECCIÓN DE INFORMACIÓN}

Cuestionario de preguntas abiertas a docentes: está conformado por 10 preguntas abiertas aplicadas de manera remota a través de un formulario, donde ellos compartieron sus opiniones. De esta manera las preguntas del $1 \mathrm{al}$ 3 permiten indagar por datos sociodemográficos (edad, experiencia y formación profesional), la pregunta 4 sobre el conocimiento del profesor acerca del modelo pedagógico que maneja la institución donde laboran, de las preguntas 5 a la 7 se quiere conocer sobre la manera en que el docente a través de sus prácticas desarrolla el pensamiento crítico de los estudiantes a partir de las habilidades contenidas en este. De las preguntas de la 8 a la 10 se busca información acerca la metodología, estrategias y planeación implementadas en sus clases.

Del mismo modo se realizó una entrevista oral con un cuestionario de 6 preguntas que indagan sobre los conocimientos y experiencias de los profesores en cuanto a pensamiento crítico y sus habilidades, así como de las estrategias aplicadas en el aula de clases con el fin de verificar y validar la información que nos habían suministrado en el cuestionario escrito.

Observación no participante: realizada a los estudiantes y profesores escogidos en la muestra. El instrumento utilizado fue el diario de campo, en la cual se recopilaron observaciones que nos permitieron identificar la incidencia de las prácticas de los docentes en el desarrollo del pensamiento crítico de sus estudiantes, manejando también las grabaciones de las clases como recurso para analizar cómo se construye conocimiento y cómo los docentes generan nuevo conocimiento.

Lectura planeación de clases: los planeadores o microcurrículos de clase que diseñan los docentes fueron otro instrumento utilizado, además de material de apoyo de los profesores como lo son las guías, tareas, talleres, evaluaciones en el aula de clases y las competencias que desarrollan durante su práctica docente; para así determinar si estas ayudan a desarrollar el pensamiento crítico de los estudiantes para llevar a cabo el proceso de enseñanza y aprendizaje de las matemáticas.

\section{RESULTADOS}

A partir de los análisis del Cuestionario de preguntas abiertas a docentes, entrevista oral, observación de las clase y lectura de planeaciones se identifican ausencias en la creación de espacios por parte del profesor donde el estudiante pueda discernir acertadamente en diferentes situaciones utilizando diversos contextos de su cotidianidad, de esta manera se logra evidenciar poca reflexión y construcción de conocimientos e ideas propias del estudiante que le permitan emitir un juicio de valor acerca de las temáticas desarrolladas en clase. De igual forma en sus planeaciones y ejecución de las clases el docente no promueve en sus estudiantes desarrollo de habilidades del pensamiento crítico limitándose a un trabajo 
magistral donde es escasa la participación e intervención continua de los estudiantes.

En cuanto a la identificación de la metodología que utiliza el profesor para facilitar el desarrollo de habilidades como interpretación, explicación, inferencia, análisis, evaluación y autorregulación en aras de la búsqueda del desarrollo de pensamiento crítico en los estudiantes, los hallazgos muestran que desde la entrevista oral el profesor lo que realiza son "ejercicios" repetitivos o ejercicios al que él llama problemas, tratando de llevarlos al contexto del estudiante lo cual considera como "diario vivir" para que el estudiante lo relacione con su vida cotidiana esto sucede con respecto al caso de un profesor. En el caso del otro profesor agrega que a partir del "aprendizaje basado en el pensamiento", el estudiante puede lograr analizar, relacionar, argumentar e inferir respecto de algún tema específico.

Simultáneamente la indagación del cómo el docente desarrolla pensamiento crítico en sus estudiantes, el profesor 1 manifiesta que lo hace cuestionando preguntas "¿cómo?, ¿por qué?, ¿cuándo?,¿Cómo hacerlo?” y con esto intenta que el estudiante desarrolle sus propios conceptos. En el caso del profesor 2 indica que la "pregunta no debe faltar" considerando que de esta forma el estudiante puede plantear y generar conocimientos nuevos,

Ambos docentes manifiestan utilizar con frecuencia actividades que fomentan la participación de sus estudiantes generando preguntas durante el desarrollo de la clase con las cuales fomentan habilidades del pensamiento crítico, teniendo en cuenta el trabajo cooperativo y colaborativo entre todos los miembros que hacen parte de la clase. Se observa que el profesor 1 para la preparación de sus clases tiene en cuenta el modelo pedagógico y lineamientos propuestos por la institución, por otro lado, el profesor 2 manifiesta que su planeación de clase la realiza teniendo en cuenta el estilo y ritmo de cada uno de los estudiantes, olvidando de esta manera un punto clave para poder desarrollar pensamiento crítico y sus respectivas habilidades.

Los profesores se mantuvieron en su opinión en lo referente a la creación de espacios en sus clases para cuestionar a los estudiantes y de esta manera poder lograr una mejor comprensión de las temáticas, indican en sus respuestas que se enfrentan a dificultades que pueden "desenfocar" la búsqueda de habilidades del pensamiento crítico.

Finalmente, con a relación a las estrategias usadas inicialmente por el profesor1 había expresado que se debía tener en cuenta el aprendizaje cooperativo y colaborativo, la resolución de ejercicios y problemas y la clase magistral, en la entrevista puntualizó que un punto clave son las preguntas problemas antes de conceptualizar, teniendo en cuenta en la planeación de sus clases el modelo pedagógico de la institución en cambio el profesor dos manifiesta utilizar estrategias vivenciales para así poder desarrollar las habilidades del pensamiento crítico en el aula de clases.

Teniendo en cuenta la Imagen 1 es importante señalar que al momento de realizar dichas observaciones Colombia se encontraba en Cuarentena Obligatoria por presencia de Covid-19. Por lo tanto, la institución se involucró a la modalidad virtual utilizando la plataforma Microsoft Teams, la cual forma parte de paquete 365 de Microsoft office. Se puede apreciar que ambos docentes no ejecutan lo señalado tanto en el cuestionario sociodemográfico como en la entrevista, mostrando de esta manera una incoherencia con respecto a sus respuestas y señalamientos; por lo tanto, es claro que las interpretaciones dadas por los docentes en el cuestionario sociodemográfico y en la entrevista que se les realizo no se relaciona directamente con su quehacer en el aula de clases ya que faltan colocar en práctica más estrategias y más 
organización al momento de la planificación de sus eventos pedagógicos. Para finalizar lo relacionado a la práctica de los profesores no estuvo encaminada al diseño de actividades y creación de espacios que generen un ambiente de trabajo que le ofrezca a sus estudiantes la manera de pensar críticamente.

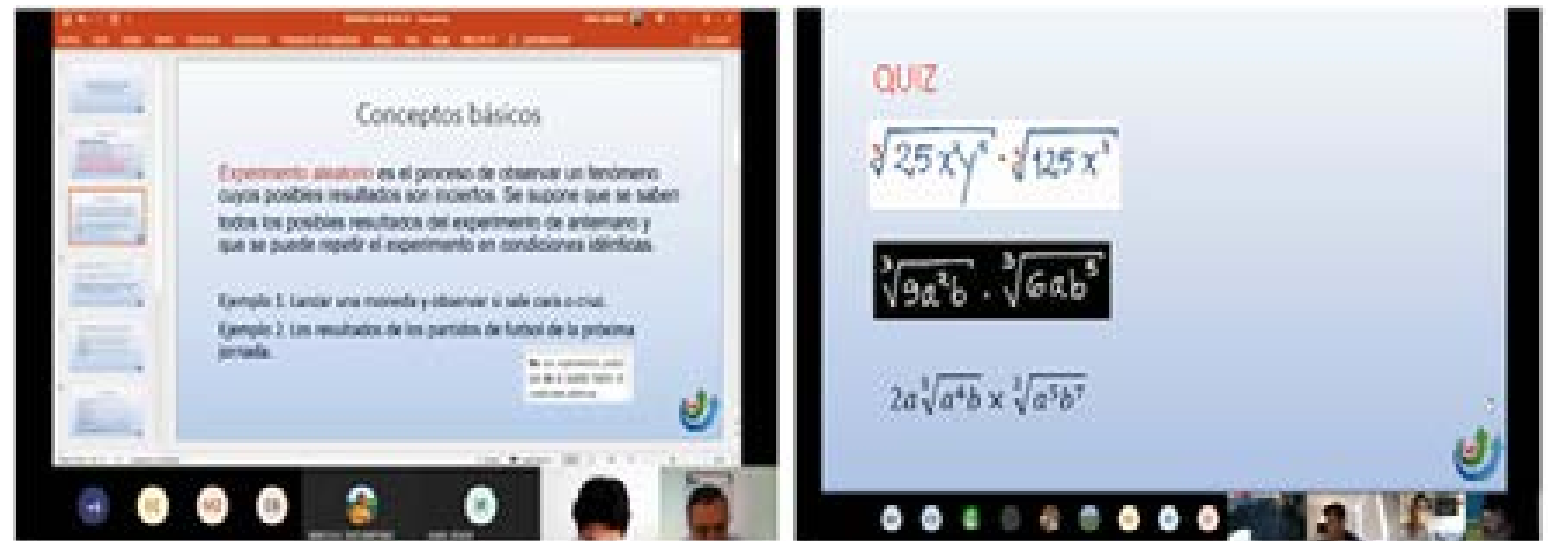

Imagen 1 Escena de la clase de los profesores en la enseñanza remota.

Fuente: De la investigación

A partir de las observaciones que se realizaron en cada una de las clases de los profesores y los aportes de los referentes Nuñez et al. (2017) y Alquichire y Arrieta (2018) no se evidencia en el desarrollo de sus clases la intención de generar espacios donde se desarrollen las habilidades del pensamiento crítico en el aula: en lo referente a cada una de estas los hallazgos dan cuenta de:

Interpretación: tanto el profesor 1 como el profesor 2 pocas veces logran que los estudiantes decodifiquen y clarifiquen los conceptos trabajados en la clase.

Análisis: esta habilidad no se evidencio en la práctica de ninguno de los dos profesores ya que no se mostró examinación de ideas, detección y análisis de argumentos, solo se limitaron a decir un concepto, a realizar ejemplos y ejercitación del algoritmo de una operación o concepto básico.

Evaluación: en este caso el profesor1 intento en algunas clases fomentar esta habilidad evaluando la credibilidad y calidad de los argumentos presentados por sus estudiantes en tareas y ejemplos, sin embargo, no logra inducir a un buen razonamiento reflexivo a sus estudiantes. Con respecto al profesor 2 la manera cómo evalúa en varias ocasiones es generando actividades de ejercitación mas no de comprensión, interpretación o análisis.

Inferencia: no hay evidencia de conjeturas frente a cualquier actividad realizada y a partir de estas no se logra observar obtención de conclusiones de ningún tipo.

Explicación: En algunos encuentros ambos profesores intentaron que sus estudiantes describieran la forma, los métodos y resultados adquiridos en sus compromisos o actividades se evidencia poca participación por parte de los estudiantes cuando se les pide que argumente o expliquen un proceso.

Autorregulación: En la práctica de ambos profesores no se incluye la auto- examinación y auto- corrección. 
Con base en el análisis de esta información desde los procesos desarrollados por los profesores en cada una de sus clases y la participación de los estudiantes en el proceso, se resalta la importancia del rol del docente al momento de incentivar cada una de las habilidades de pensamiento crítico descritas anteriormente. A partir de cada una de las observaciones realizadas se plantea una propuesta de secuencias didácticas donde se incluyen cinco actividades, presentadas a los dos profesores de séptimo y noveno, validándose la apropiación y desarrollo del pensamiento crítico en el aula de clases desarrollar habilidades de pensamiento y poder lograr desarrollar competencias básicas en los estudiantes.

En la actividad 1, se generan espacios donde el estudiante pueda comprender y expresar el significado de una situación específica, de esta forma se busca promover la interpretación y el análisis mediante un conjunto de situaciones problemas que se toman como ideas previas (Imagen 2), el docente realiza cuestionamientos que permitirán que el estudiante participe activamente y reflexione sobre las situaciones presentadas y pueda inferir y tener buenos argumentos en sus conclusiones planteadas.

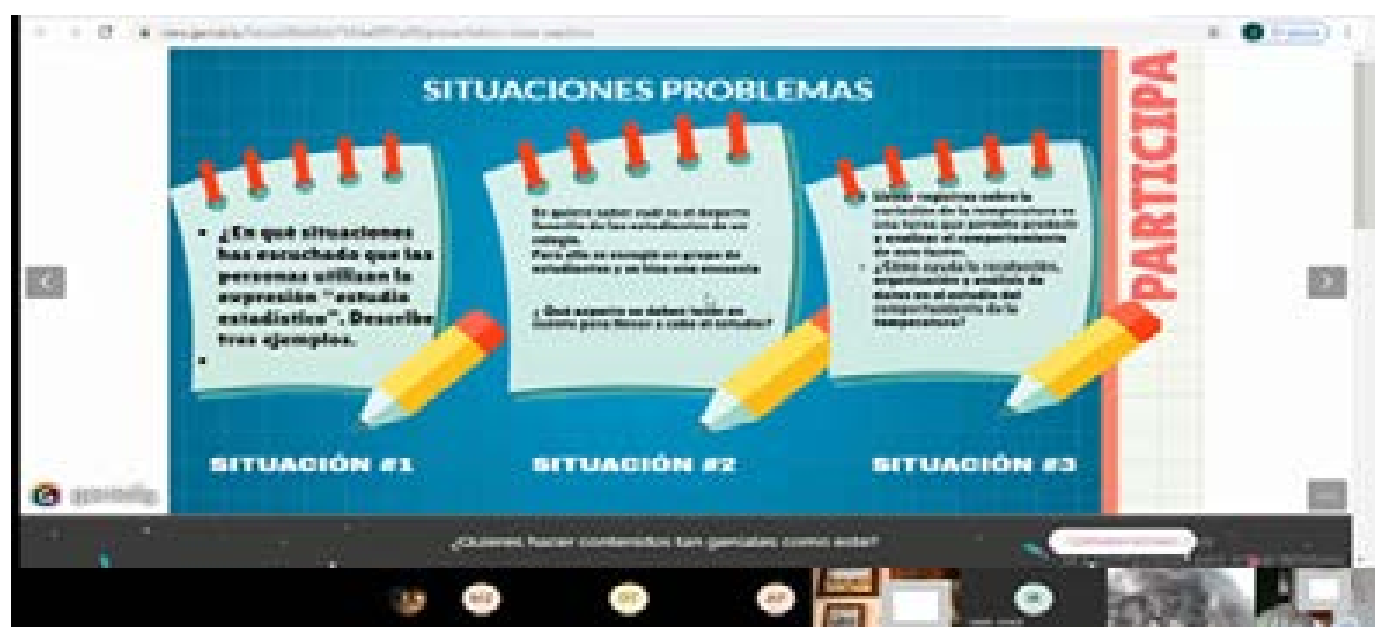

Imagen 2 Situaciones de aprendizaje propuestas clases remota

Fuente: De la investigación

En la actividad 2 mediante preguntas y videos (Imágenes 3 y 4 el docente busca que el estudiante pueda identificar correctamente los datos necesarios para poder lograr conclusiones a partir de los conceptos, lo que permitirá que pueda inferir y explicar de manera acertada los resultados adquiridos.

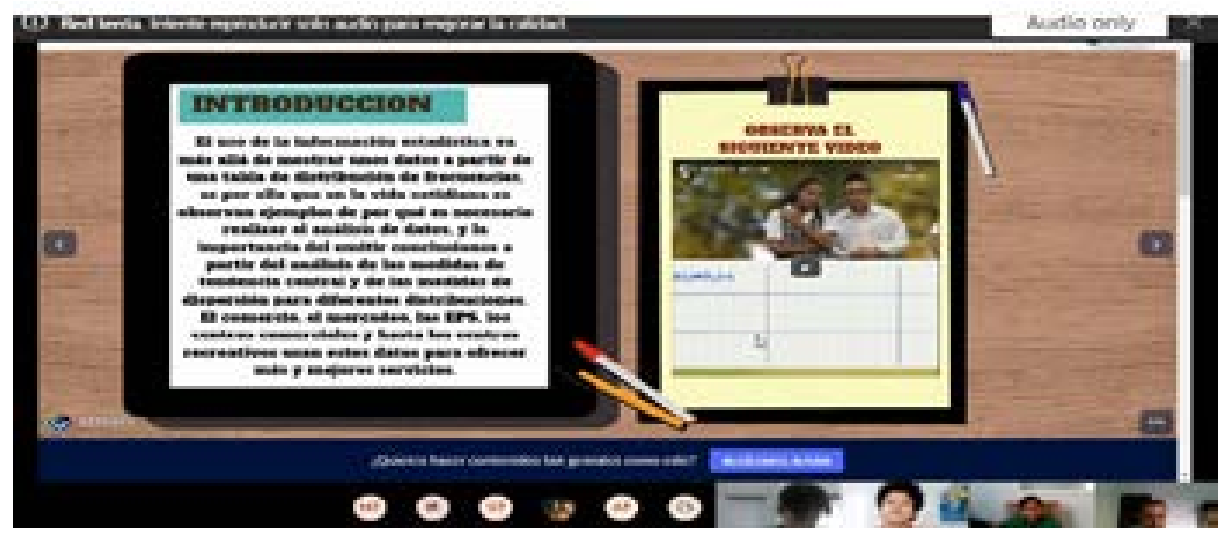

Imagen 3 Actividad en clases remota

Fuente: De la investigación 
En la actividad 3 y 4 , el docente será un guía en el proceso de evaluación y calidad de argumentos de sus estudiantes por medio de una serie de situaciones problemas donde permitirá que se cuestione la credibilidad de las respuestas de sus compañeros y de esta manera llegar a que estos logren evaluar e inferir diferentes representaciones y puntos de vistas (Imagen 4).

Con la actividad 5 se pretende que el estudiante emplee habilidades de análisis y evaluación luego que el docente lo lleve a la interpretación de lo conocido y desconocido permitiendo de esta manera a través de interrogantes lograr que el estudiante se auto-examine y auto-corrija sus propios resultados.

Teniendo en cuenta, la planeación, organización, seguimiento y virtualidad de las clases se diseñó un plan de clases para séptimo grado el cuál fue compartido con el profesor1 para que este ejecutará en la temática de medidas de tendencia central. Estas clases fueron diseñadas con el fin de incentivar la participación constante del estudiante, el desarrollo de habilidades del pensamiento crítico y construcción del conocimiento. En el diseño de estas secuencias de actividades se tuvo en cuenta plantear acciones que permitan el mejoramiento del aprendizaje, para ello se siguió la propuesta del Ministerio de Educación Nacional en Colombia, a través de una caja de materiales que se proponen en el documento llamado Siempre E; donde se propone tres momentos específicos que se relacionan en las orientaciones pedagógicas se implementó en la clase del profesor1, un espacio de reflexión para llevar acabo el diseño de la clase, la ejecución y análisis de las actividades que se lograron generar en este espacio.

El primer momento de esta clase se motiva a los estudiantes a dar a conocer sus saberes previos motivándolos frente a la temática de medidas de tendencia central, los cuáles se pueden desarrollar mediante preguntas enfocadas a situaciones problemas con el fin de que se involucren de manera constante y pueda justificar y comprender sus aportes y el de sus compañeros. Con estos interrogantes el docente tiene la oportunidad de conocer los conocimientos previos y la comprensión por parte de los estudiantes de la temática a tratar y de esta manera visionar el logro del aprendizaje propuesto.

Momento de estructuración y práctica: Para este momento de la clase se proponen actividades de aprendizajes diversas con el fin de que el estudiante, observe, interprete, comprenda, analice y logre identificar un concepto a partir de su propia reflexión. En esta parte de la clase el docente será un orientador y realizará intervenciones en momentos específicos para aclarar la conceptualización de cada una de las medidas de tendencia central (moda, media o promedio y mediana) o comentar alguna participación de sus estudiantes,

En las actividades de aprendizaje propuestas ( $\mathrm{N}^{\circ} 1, \mathrm{~N}^{\circ} 2, \mathrm{~N}^{\circ} 3 \mathrm{Y}^{\circ} \mathrm{N}^{\circ}$ ) a partir de la observación del contenido proyectado y compartido en pantalla; se tiene como finalidad que el grupo en general, pueda reconocer ¿cuántas? y ¿cuáles? son las medidas que se utilizan para describir el comportamiento de un conjunto de datos cuantitativos. En este sentido crear un ambiente de participaciones constantes, donde se despierte el sentido analítico y común de los estudiantes, así como la conexión concreta e intervenciones relacionadas directamente con el tema en discusión (Imagen 4); para el caso la Moda, Media o promedio y Mediana. 


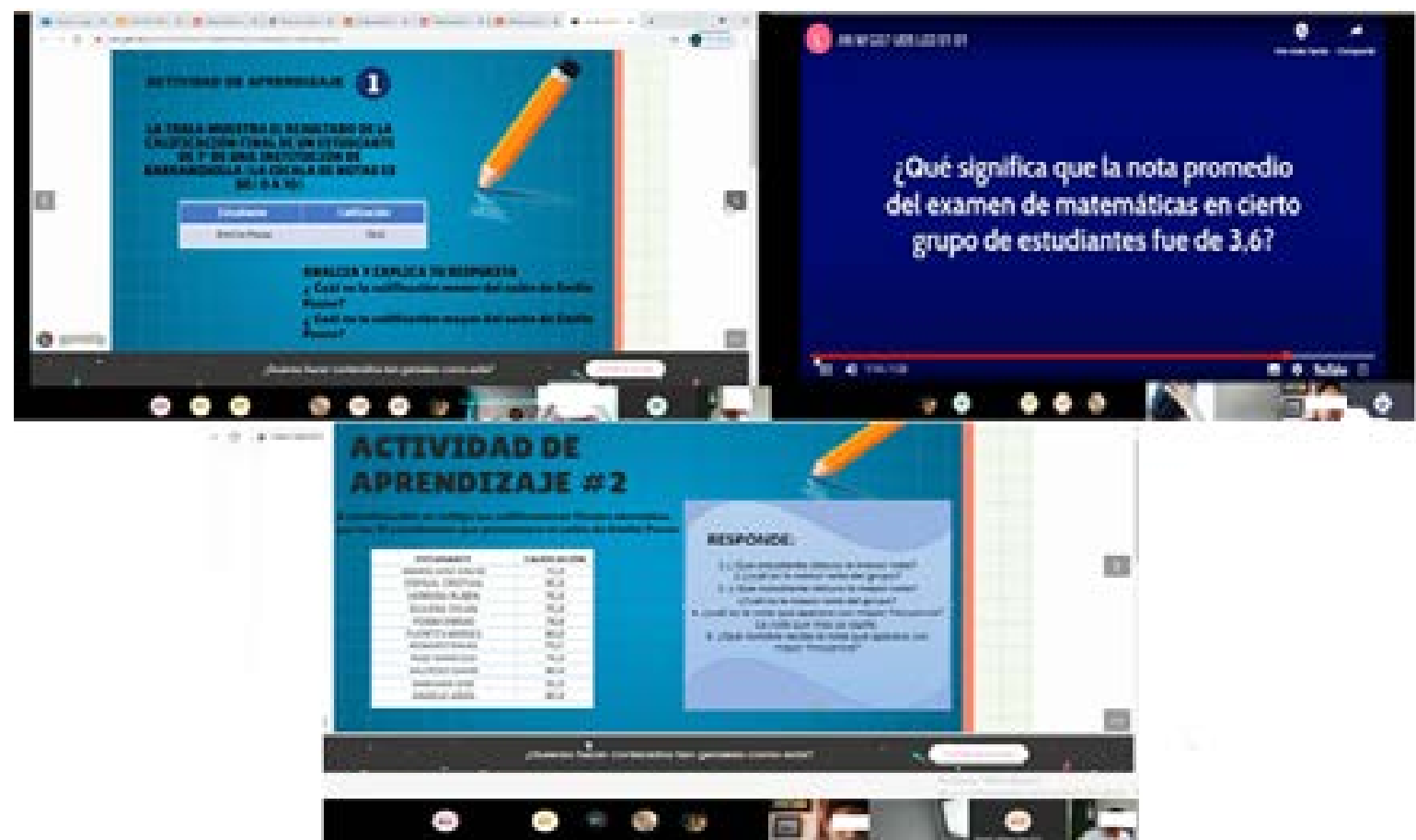

Imagen 4 Actividades de aprendizaje clases remotas

Fuente: De la investigación

En cada una de estas actividades, se encuentran ejercicios prácticos donde se ejercitará la parte procedimental de cada medida, luego de haber comprendido y logrado por ellos mismo conceptualizar la temática.

Momento de transferencia y valoración: Finalmente, para culminar la clase el docente debe formular preguntas y situaciones problemas a sus estudiantes con el fin de indagar la claridad de estos con respecto al objetivo de aprendizaje. De esta manera expresa curiosidad por conocer lo aprendido, en lugar de responder mecánicamente a sus estudiantes. La sección se cierra asignando una actividad de aprendizaje que realizar en la casa; para colocar en práctica lo visto y desarrollado.

Los resultados obtenidos en esta investigación permiten inferir que al utilizar las secuencias didácticas no se adquiere por completo pensamiento critico ya que debe ser constante, pero si se favorece el desarrollo de las habilidades del pensamiento tales como interpretación, análisis, explicación, inferencia, evaluación, autorregulación a través de actividades (Imagen 5) que generen e involucren al estudiante activamente.

\section{Imagen 5 Actividades de aprendizaje clases remotas}

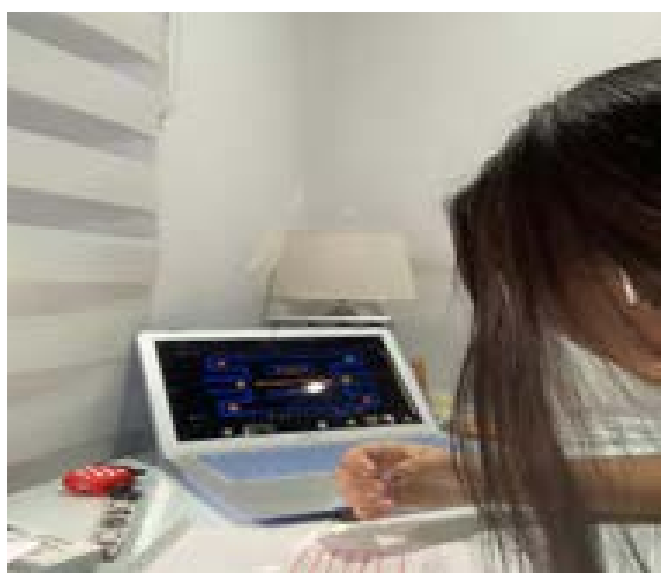

Fuente: De la investigación

\section{DISCUSIÓN}

Dados que los efectos que se identificaron fueron aumento en las habilidades del pensamiento crítico tales como la interpretación, análisis, inferencia, evaluación, explicación y autorregulación, se observó la modificación 
del discurso de los profesores, su actuación e interacción en el aula, lo cual se relaciona con lo planteado por Tamayo et al. Zona y Loaiza (2015) cuando se refieren a que el actuar del maestro en el aula de clases incide directamente en el desarrollo del pensamiento crítico de los estudiantes, complementando la importancia del rol del maestro y la didáctica de su practica en el proceso de enseñanza.

Teniendo en cuenta que para fortalecer la didáctica del pensamiento crítico y sus habilidades es indispensable pensar en un aprendizaje activo que permita la construcción del conocimiento (Paul y Elder 2008), manifiestan la importancia que tiene el asimilar las temáticas o conceptos iniciales para que posteriormente se pueda aplicar y analizar el valor de los conocimientos adquiridos, de ahí la importancia que le damos al papel del docente en cuanto a la creación de espacios y estrategias que permitan promover este tipo de pensamiento, por lo tanto es indispensable (Facione 2007) incentivar un valor crítico, que inicia de un proceso de exploración de la curiosidad, agudeza mental, adquisición de información fiable y confiable para que la meta de enseñanza pueda favorecer al aprendizaje de las matemáticas y el desarrollo de las habilidades del pensamiento crítico.

Es importante señalar que el pensamiento crítico se debe despertar e incentivar constantemente, en el caso de ambos docentes cada uno desde su temática a trabajar realiza preguntas a sus estudiantes sin generar ningún tipo de reflexión crítica y de esta manera no se logra involucrar a todos sus estudiantes conectados en línea, con esto es importante señalar a Merchán (2012), Camargo y Useche (2015) cuando menciona que para fomentar el pensamiento crítico se deben generar preguntas debido a la relevancia que tienen como herramientas especificas en el proceso de enseñanza-aprendizaje.

Siendo así, en el diseño de secuencias didácticas se requiere que involucren interrogantes que conlleven y le exijan a los estudiantes la capacidad de discernir, analizar, evaluar, inferir, comprender y sobre todo le permita profundizar en la búsqueda de nueva información. De esta manera se hace necesario plantear preguntas que no sean solo de evaluación de teoría; es decir, se debe buscar que el estudiante verifique sus argumentos utilizando fuentes claras $y$ precisas que le ayuden a dejar de un lado el uso constante del libro o cuaderno y así pueda generar operaciones mentales que le ayuden a crear y descubrir nuevos conocimientos.

En todo este proceso juega vital importancia la planeación que realiza el docente para implementar en sus clases, señalando en este punto a Chongy Marcillo (2020) refiriendose a que las actividades deben ser innovadoras recomiendan cambiar lo tradicional por contenidos que permitan obtener un aprendizaje eficaz tal como se busca con las secuencias didacticas planteadas en esta investigación; resaltando que en la búsqueda del desarrollo de habilidades del pensamiento crítico se genereraron cinco actividades y que no solo con la implementacion de estas se desarrolla completamente, recordemos que debe ser permanente, constante y dinamica para poder tener un impacto general.

Este articulo presenta contribuciones positivas para el contexto educativo en el cual se encuentran vinculados los profesores de matemática y los estudiantes de séptimo y noveno grado de una institución privada de Barranquilla, pues les permite identificar y potencializar aspectos sobre fortalecimiento de habilidades del pensamiento crítico desde su praxis en el aula de clases.

\section{CONCLUSIONES}

Es de vital importancia que en el aula de clase se conciba un ambiente reflexivo, crítico y confortable por parte de los docentes y de sus estudiantes. Por lo tanto, es fundamental 
realizar una excelente preparación y planeación de actividades que generen la construcción de un conocimiento integral donde se involucre de manera activa en el proceso de enseñanza y aprendizaje las participaciones del estudiante y docente; para que de esta manera se pueda generar una mejor comprensión y asimilación de las temáticas que se quieren trabajar en el aula de clase y se logren los objetivos y aprendizajes esperados.

El objetivo general de la presente investigación fue caracterizar las practicas del docente de matemática en su incidencia en el desarrollo del pensamiento crítico de los estudiantes. Los efectos fueron notables a través de las observaciones que se realizaron durante todo el proceso investigativo donde se pudo percibir cambios en las estrategias y planeaciones realizadas por los docentes.

Los resultados aportados en esta investigación dan muestra de lo necesario que es vincular de manera clara y seguida al estudiante con el desarrollo del pensamiento crítico y sus habilidades (interpretación, análisis, inferencia, explicación, evaluación, autorregulación) desde la práctica del docente en este caso en el quehacer del docente de matemáticas. Por lo tanto, aplicar el diseño de estas tareas didácticas en matemáticas posibilita la asimilación del aprendizaje y a la innovación por parte de los docentes de salir de la rutina y proponer nuevos ambientes donde la participación de sus estudiantes sea activa y permanente durante la clase.

\section{REFERENCIAS}

Aguilar, L., Alcántara, I., \& Braun, A. (2020). Impacto del Pensamiento Crítico en las habilidades para el campo laboral. Revista de investigación en ciencias sociales y humanidades, Vol. $7 \mathrm{~N}^{\circ} .2$, 2020, págs.166-174.
Alquichire,S. L., \& Arrieta J. C. (2018). Relación entre habilidades de pensamiento crítico y rendimiento académico. Voces y Silencios: Revista Latinoamericana de Educación, Vol. 9 Nº. 1, 2018, págs.2852.

Álvarez,C \& San Fabian J. L. (2012). La elección del estudio de caso en investigación educativa. Gazeta de Antropología, Vol. $1, N^{\circ} .28,2012$, págs.1-12.

Arévalo,V.E., (2015). ¿Cómo se enseñan las matemáticas en la escuela primaria? XIV CIAEM - IACME. Conferencia Interamericana de Educación Matemática, págs.1-12. Chiapas, México.

Ayola,M. Y. \& Moscote E. M. (2018). Pensamiento Crítico, estrategias para estimularlo e incidencia en la práctica pedagógica en el programa de licenciatura en educación infantil de la Univeridad de la Guajira. Revista Boletín Redipe, Vol. 7 $N^{\circ} .10,2018$, págs. 147-166.

Camargo, L., \& Useche, J. (2015). Las preguntas como herramientas intelectuales para el desarrollo de un pensamiento crítico. Revista electrónica de Humanidades, Educación y Comunicación Social, $N^{\circ}$. 20, 2015, págs.145-156.

Campos, D. R. (2020). Pensamiento crítico y el aprendizaje de la matemática en estudiantes ingresantes a la universidad. Revista EDUSER, Vol. 7, N.2, 2020, págs. 82-94.

Cangalaya, S. L. (2020). Habilidades del pensamiento cítico en estudiantes univeritarios a través de la investigación. Desde el Sur, Vol.12, №.1, 2020, págs. 141-153. 
Caradonna, L. (2017). Aprendizaje significativo: Felicidad, motivación y estrategias de los docentes. Tesis Doctoral.

Chong-, P. G. \& Marcillo,C.E. (2020). Estrategias pedagógicas innovadoras en entornos virtuales de aprendizaje. Dominio de las Ciencias, Vol.6, Nº.3, 2020,págs 56-77.

Facione, P. (2007). Pensamiento Crítico:¿Qué es $y$ por qué es importante? Obtenido de Versión 2007 en Español: http://www.eduteka.org/ PensamientoCriticoFacione.php

Febres C , M., Alirio P.Á \& Africano G.B. (2017). Las pedagogías alternativas desarrollan el pensamiento crítico. Educere, La Revista Venezolana de Educación, Vol.21, N.69, 2017, págs. 269-274.

Frías G.M., Haro Á, Y. \& Artiles O. I. (2017). Las habilidades cognitivas en el profesional de la Información desde la perspectiva de proyectos y asociaciones internacionales. Investigación Bibliotecológica, Vol.31, N.71, 2017, págs.201-218.

Gavilanes, E. G.\& Benavides-S,J. (2018). Desarrollo Del Pensamiento Crítico en los estudiantes a traves de del proceso de enseñanza- aprendizaje de la asignatura lengua y literatura. ROCA. Revista científico - educacional de la provincia Granma., Vol.14, $N^{\circ} .1,2018$, págs. 2-11.

Jiménez E. A, Limas, L.J. \& Alarcón, J. E. (2016). Práticas Pedagógicas Matemáticas de Profesores de una Institución Educativa de Enseñanza Básica y Media1. Praxis\&Saber, Vol.7, $N^{\circ} .13,2016$, págs 127-152.
Llinares, S. (2011). Tareas matemáticas en la formación de maestros.Caracterizando perspectivas. Revista de Didáctica de las Matemáticas, Vol.78, 2011, págs, 5-16.

López A,G. (2012). Pensamiento crítico en el aula. Revista de la Escuela Universitaria de Magisterio de Toledo, $N^{\circ} .22,2012$, págs, 41-60.

López Q,G. (2014). La ensenañza de las matemáticas, un reto para los maestros del siglo XXI. Praxis Pedagógica $N^{\circ} .15$, 2014, págs,55-76.

Mackay C, R., Franco C. D \& Villacis, P. W. (2018). El pensamiento crítico aplicado a la investigación, Universidad $y$ Sociedad, Vol.10, $N^{\circ} .1,2018$, págs, 336-342.

Merchán Price, M. S. (2012). Cómo desarrollar los procesos del pensamiento crítico mediante la pedagogía de la pregunta. Actualidades Pedagógicas, Vol.1, $N^{\circ} .59,2012$, págs.119-146.

Miranda, C. (2003). El Penamiento Crítico En Docentes De Educación General Básica En Chile: Un Estudio De Impacto. Estudios Pedagógicos N²9, 2003, págs.39-54.

Nuñez, S., Ávila, J., \& Olivares, S. (2017). El desarrollo de pensamiento crítico en estudiantes universitarios por medio de aprendizaje basados en problemas. Revista iberoamericana de educación superior, Vol.8, $N^{\circ} .23,2017$,págs. 84103.

Parra F, K. (2014). El docente y el uso de la mediación en los procesos de enseñanza y aprendizaje. Revista de Investigación, Vol. 38, N.83, 2014, págs.155-180. 
Richard, P. \& Linda. E. (2003). La mini guía para el pensamiento crítico: conceptos y herramientas. EEUU: Fundación para el pensamiento crítico. Recuperado el 26 de septiembre de 2020, de http://www. criticalthinking.org/resources/PDF/SPConceptsandTools.pdf

Rodríguez, M. E. (2013). La educación matemática en la con-formación del ciudadano. TELOS. Revista de Estudios Interdisciplinarios en Ciencias Sociales, Vol. 15, №.2, 2013, págs. $215-230$.

Tamayo A., O. E., Zona, R., \& Loaiza Z, Y. E. (2015). El pensamiento crítico en la educación. Algunas categorías centrales en su estudio. Revista Latinoamericana de Estudios Educativos(Colombia), Vol. 1, No.2, 2015, págs.111-133.

Yasaldez , E., Osorio, L., \& Zuluaga, L. (2018). El desarrollo de pensamiento crítico en ciencias naturales con estudiantes de básica secundaria en una Institución Educativa de Pereira - Risaralda. Diálogos sobre investigación. Temas actuales de investigación educativa, Vol.9, $N^{\circ} .6,2018$, págs. 1-24. 